\author{
Cun Zhang, Lei Chen, Shaohua Chen
}

\title{
Adhesion between two radially collapsed single-walled carbon nanotubes
}

Received: 27 January 2013 / Revised: 28 April 2013 / Published online: 31 May 2013

(C) Springer-Verlag Wien 2013

\begin{abstract}
Continuum mechanics analysis and molecular mechanics simulations are performed to study adhesion between two identical, radially collapsed single-walled carbon nanotubes. Not only the inter-adhesion energy between nanotubes but also the inner adhesion energy in a nanotube is considered. A closed-form solution to the adhesion configuration is achieved, which is well consistent with our molecular mechanics simulation. Comparing the potential energy of the adhesion structures formed by two identical single-walled carbon nanotubes, three types of configurations, i.e., circular, deformed, and collapsed shape, will be formed with increasing carbon nanotubes radius and separated by two critical radii of the single-walled carbon nanotube. Furthermore, it is found that the collapsed adhesion structure possesses the highest interfacial adhesion energy. The results demonstrate that, as a potential application in carbon nanotube reinforced composites, arrays formed by collapsed carbon nanotubes will be optimal due to the strong interface strength.
\end{abstract}

\section{Introduction}

During the past two decades, carbon nanotubes (CNTs) as a novel nanostructured material are attracting much attention. As mentioned by Glassmaker and Hui [1], electrically, single-walled carbon nanotubes (SWNTs) are one-dimensional conductors or are semiconductors with very few surface states. Mechanically, the atomic structure has very few flaws or dislocations and is held together by strong, covalent bonds between carbon atoms. Thermally, phonon transfer dominates heat conduction. The phonon mean-free path in SWNTs is long compared with traditional materials due to fewer structural defects and a lack of phonon scattering sites.

In order to realize the potential applications of CNTs, the first step is to produce single CNTs or aligned CNT arrays with stable configurations. For a SWNT, a natural equilibrium configuration has a circular cross section. However, during the process of producing CNTs, interactions of the microscale force in a nanotube or among neighboring nanotubes arise [2-5]. The van der Waals (vdW) interactions in a CNT or among CNTs play a dominant role due to their small sizes [6], which is usually called adhesion mechanics of nanotubes. Sometimes, adhesion will show a great effect on the geometry and stability of an individual SWNT [7]. The circular cross section can be deformed by vdW interactions between carbon atoms in a single CNT or those among CNTs, leading to different equilibrium configurations, which is called self-deformation. The reason is that CNTs capture the characteristics of hollow cylindrical structures, which render them susceptible to lateral deformation. During the process of producing SWNTs or aligned SWNT arrays, it was found that SWNTs with small diameters can maintain a cylindrical or honeycomb structure [6]. When the diameters of CNTs become larger and larger, self-deformation will happen.

C. Zhang $\cdot$ L. Chen $\cdot$ S. Chen $(\varangle)$

LNM, Institute of Mechanics, Chinese Academy of Sciences,

Beijing 100190, China

E-mail: chenshaohua72@hotmail.com

Tel.: 86-10-82543960

Fax: 86-10-82543977 
An interesting collapsed structure of CNTs was first observed and explored by Ruoff et al. [7] and Chopra et al. [8] using transmission electron microscopy (TEM). Many other researchers also found such a collapsed structure using atomic force microscopy (AFM) later [9-11]. The cross section of a collapsed structure consists of two parts: flat regions like two adhering graphenes in the middle and curved regions like buckyballs at each end, which was called a collapsed nanotube [8], flattened nanotube [12], or closed-edged graphene nanoribbon [13]. Such a collapsed shape has been widely observed in the synthesis of large-diameter CNTs [14-16], applications of CNT fibers [17-19], CNT/polymer composites [20,21], and CNT nanoactuators [22]. Recently, CNT bundles containing large numbers of collapsed CNTs could be grown from preformed $4.0 \mathrm{~nm}$ diameter aluminum-iron oxide particles directly [13].

When CNTs jump from a circular configuration to a collapsed one, their properties will change dramatically. For example, the collapsed configuration can significantly alter the electronic behavior of CNTs [23-26], and the collapsed CNTs will possess a high electrical conductivity [27]. In the aspect of mechanical properties, self-collapse can increase the strength of CNT bundles up to a value of about $30 \%$ compared to a circular configuration [28]. Furthermore, Zhang and Li found that self-collapse could improve the inter-tube frictional force by a factor of about 1.5-4 [29].

Since self-deformation and self-collapse of CNTs are mainly due to vdW interactions in a CNT or between CNTs, adhesion mechanics of nanotubes attracts many researchers' interests, especially the adhesion problem between nanotubes.

Lots of work on the adhesive interaction between CNTs has been carried out. For example, Chen et al. estimated experimentally the binding energy of parallel CNTs and the geometry parameters from TEM images of two bonded CNTs [30]. Using the Lennard-Jones potential and smeared-out approximation, the interaction energies between two crossed SWNTs with infinite length and different radii have been studied [31-34]. Tang et al. analyzed successfully the adhesion between two identical SWNTs with the continuum mechanics and molecular simulations [35]. A critical radius of the SWNT was found, below which the contact area between the two SWNTs is zero. However, only the adhesion problem of two self-deformed SWNTs was investigated in Tang et al. [35]. What will happen between two adhesive self-collapsed SWNTs since such an adhesive behavior is often found during fabrication of CNTs? What will influence the detailed configuration of two adhesive self-collapsed SWNTs? What is it about the interfacial adhesion energy of the self-collapsed configuration compared to the adhesive self-deformed one?

In order to answer the above questions, the adhesion between two identical, radially collapsed SWNTs is studied in this paper based on the continuum analysis and molecular simulations, in which the coupling effects of self-collapse and inter-tube interaction are considered in the theoretical model.

\section{The adhesive configuration of two self-collapsed SWNTs}

The collapsed morphology of a SWNT consists of a flat zone in the middle part and two noncontact regions at the ends, which has been successfully predicted theoretically by Tang et al. [35] and Liu [36]. Good agreements are achieved between the theoretical analysis and molecular simulations. Figure 1a shows an elastica model to study the adhesion of two identical radially collapsed SWNTs, each of which has an initial radius $R$. During the analysis, SWNTs are assumed to be inextensible. After adhesion, each tube is still composed of a flattened contact zone in the middle and two noncontact regions at the ends, but the configuration of each tube, compared to the single collapsed SWNT, changes due to the vdW interaction between the upper and lower tubes as shown in Fig. 1a. The contact widths due to the self-adhesion and inter-tube adhesion are $2 a$ and $2 b$, respectively. Normally, the vdW force between two carbon atoms is repulsive at a very close range, so both the distance between the upper edge and the lower one of a SWNT and the distance between two collapsed SWNTs are defined by an equilibrium separation $d_{0}$, i.e., $d_{1}=d_{0}$ and $d_{2}=d_{0}$ as shown in Fig. 1a. Due to the symmetry of the structure, only a quarter of the structure will be analyzed, as shown in Fig. 1b. In addition to the Eulerian coordinates $(x, y)$, the arc length $s$, as a Lagrangian coordinate, is also adopted as a variable in the analysis. $\phi$ denotes the slope angle, whose values at some typical points are given in Fig. $1 \mathrm{~b}$.

The inextensible condition gives

$$
a+b+l=\pi R,
$$

where $l$ is the total arc length of the noncontact region at the end.

The geometric equations and boundary conditions are expressed as

$$
\dot{x}=\cos \phi, \quad \dot{y}=\sin \phi,
$$


(a)

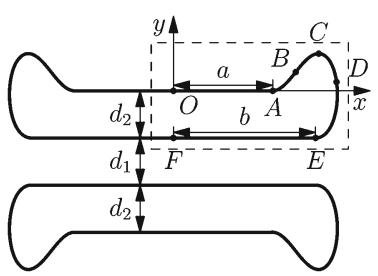

(b)

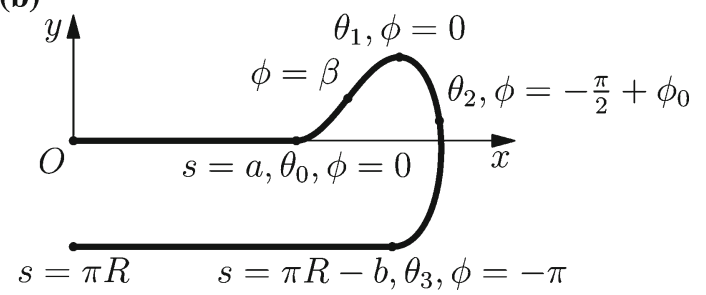

Fig. 1 Two identical, radially collapsed nanotubes in adhesive contact. a Adhesive collapsed configuration. b A quarter of the adhesion structure for theoretical analysis

$$
\begin{gathered}
x(a)=a, \quad y(a)=0, \quad \phi(a)=0, \\
x(\pi R-b)=b, \quad y(\pi R-b)=-d_{2}, \quad \phi(\pi R-b)=-\pi,
\end{gathered}
$$

where the dot above a parameter stands for its derivative with respect to the arc length $s$ and Eqs. (2) denote the kinematic relations.

The potential energy of the adhesion system is

$$
\begin{gathered}
\Pi=2 L \int_{a}^{\pi R-b}\left[D \dot{\phi}^{2}+\lambda_{1}(\dot{x}-\cos \phi)+\lambda_{2}(\dot{y}-\sin \phi)\right] \mathrm{d} s \\
+4 \pi R L u_{g}+U_{\mathrm{vdW}}^{n c}-4 a L W_{1}-2 b L W_{2}, \\
W_{1}=W\left(d_{1}+d_{2}\right)+W\left(d_{2}\right), W_{2}=W\left(d_{1}\right),
\end{gathered}
$$

where the first term on the right side of Eq. (5) is the elastic strain energy, and $L$ is the length of each tube in the out-of-plane direction. $D$ is the effective bending stiffness of a flat graphite sheet, which has been found to be insensitive to the chirality of the nanotubes [35] and is usually achieved from molecular simulations. The effective bending stiffness obtained from our molecular mechanics simulations with AIREBO potential (without torsion term) is $D=1.33 \mathrm{eV}$, which is consistent with that in Lu et al. [37]. $\lambda_{1}$ and $\lambda_{2}$ are two Lagrange multipliers, enforcing the additionally geometrical relations of Eq. (2). The second term on the right side $4 \pi R L u_{g}$ is the graphite sheet formation energy, which is a constant. The third term $U_{\mathrm{vdW}}^{n c}$ is the external $\mathrm{vdW}$ energy produced by the interactions between the two nanotubes outside the contact region, which is also assumed to be a constant independent of the geometry [35]. The fourth and the last terms are the adhesion energies among the four flat regions, in which the interaction energy between the flat region with length $2 a$ of the upper tube and the one of the lower tube is neglected due to a relatively small magnitude.

The adhesion energy per unit area $W$ equals to the work per unit area to separate two parallel graphite sheets from a distance $d$ to infinity, which can be obtained via the integration of the Lennard-Jones potential as

$$
W(d)=-2 \pi \epsilon \rho^{2} \sigma^{2}\left[\frac{2}{5}\left(\frac{\sigma}{d}\right)^{10}-\left(\frac{\sigma}{d}\right)^{4}\right]
$$

where $\rho=0.38 \AA^{-2}$ is the number of carbon atoms per unit area, $\epsilon$ is a parameter determining the depth of the potential well, and $\sigma$ is a length scale parameter that determines the position of the minimum potential. 
Minimizing $W(d)$ provides an equilibrium separation $d_{0}=\sigma$. In this paper, we take $\epsilon=2.84 \mathrm{meV}$ and $\sigma=3.4 \AA$. Then, according to Eqs. (6) and (7), we have $W_{1}=19.72 \mathrm{meV} / \AA^{2}$ and $W_{2}=17.87 \mathrm{meV} / \AA^{2}$.

Letting the variation of the potential energy function of Eq. (5) be zero, i.e., the principle of the minimum potential energy,

$$
\delta \Pi=\delta \Pi_{1}+\delta \Pi_{2}=0
$$

where

$$
\begin{gathered}
\delta \Pi_{1}=2 L \int_{a}^{\pi R-b}\left[-\left(2 D \ddot{\phi}-\lambda_{1} \sin \phi+\lambda_{2} \cos \phi\right) \delta \phi+(\dot{x}-\cos \phi) \delta \lambda_{1}\right. \\
\left.+(\dot{y}-\sin \phi) \delta \lambda_{2}\right] \mathrm{d} s+2 L \lambda_{1}(\delta b-\delta a), \\
\delta \Pi_{2}=2 L\left\{D[\dot{\phi}(a)]^{2}+\lambda_{1}-2 W_{1}\right\} \delta a+2 L\left\{D[\dot{\phi}(\pi R-b)]^{2}-\lambda_{1}-W_{2}\right\} \delta b .
\end{gathered}
$$

Then, we have

$$
\begin{gathered}
2 D \ddot{\phi}-\lambda_{1} \sin \phi+\lambda_{2} \cos \phi=0, \\
D[\dot{\phi}(a)]^{2}-2 W_{1}=0, \\
D[\dot{\phi}(\pi R-b)]^{2}-W_{2}=0 .
\end{gathered}
$$

Equation (11) is consistent with that obtained by Liu [36], where a single collapsed CNT model was analyzed. From the governing equation (11), the Lagrange multipliers $\lambda_{1}$ and $\lambda_{2}$ can be identified as the horizontal force and the vertical force, respectively.

Using Eqs. (12) and (13), we can simplify Eq. (11) as

$$
D \dot{\phi}^{2}=C_{1}-\lambda_{1} \cos \phi-\lambda_{2} \sin \phi
$$

where

$$
C_{1}=W_{1}+\frac{1}{2} W_{2}, \quad \lambda_{1}=-W_{1}+\frac{1}{2} W_{2},
$$

and $\lambda_{2}$ is still unknown.

Introducing parameters $\phi_{0}, C$, and $\alpha$, and letting $\sin \phi_{0}=-\frac{C \lambda_{1}}{C_{1}}, \cos \phi_{0}=\frac{C \lambda_{2}}{C_{1}}$ and $\alpha=\sqrt{\frac{C_{1}}{2 D C}},(0 \leq$ $\phi_{0} \leq \frac{\pi}{2}$ ), the governing equation (14) can be transformed into a standard form:

$$
\frac{1}{2} \dot{\phi}^{2}=\alpha^{2}\left[C-\sin \left(\phi-\phi_{0}\right)\right] .
$$

Because the increment of the arc length $\mathrm{d} s$ is always positive and the one of the slope angle is not monotonic, Eq. (16) can be further simplified as

$$
\alpha \mathrm{ds}=\frac{|\mathrm{d} \phi|}{\sqrt{2\left[C-\sin \left(\phi-\phi_{0}\right)\right]}} .
$$

For convenience of integration, another variable $\theta$ is introduced, which is related to $\phi$ by the following equation:

$$
(1+C) \sin ^{2} 2 \theta=2 k^{2} \sin ^{2} 2 \theta=1+\sin \left(\phi-\phi_{0}\right) \quad(0 \leq \theta \leq \pi, \quad k>0),
$$

where

$$
C=2 k^{2}-1
$$


Then, we have

$$
\begin{gathered}
\phi_{0}=-\arcsin \frac{\lambda_{1}\left(2 k^{2}-1\right)}{C_{1}}, \\
\cos \left(\phi-\phi_{0}\right)=2 k \sin 2 \theta \sqrt{1-k^{2} \sin ^{2} 2 \theta} \\
|\mathrm{d} \phi|=\frac{4 k|\cos 2 \theta|}{\sqrt{1-k^{2} \sin ^{2} 2 \theta}} \mathrm{d} \theta \\
\sqrt{2\left[C-\sin \left(\phi-\phi_{0}\right)\right]}=2 k|\cos 2 \theta|,
\end{gathered}
$$

and

$$
\frac{|\mathrm{d} \phi|}{\sqrt{2\left[C-\sin \left(\phi-\phi_{0}\right)\right]}}=\frac{2 d \theta}{\sqrt{1-k^{2} \sin ^{2} 2 \theta}} .
$$

The angle $\theta$ at the point of $x=a$ and $y=0$ is defined as $\theta_{0}$, which can be obtained as follows due to $\phi=0$ :

$$
\theta_{0}=\frac{1}{2} \arcsin \frac{1}{k} \sqrt{\frac{1-\sin \phi_{0}}{2}}
$$

At the point of $x=b$ and $y=-d_{0}, \theta$ is defined as $\theta_{3}$. Then, $\phi=-\pi$ yields

$$
\theta_{3}=\frac{1}{2}\left[\pi+\arcsin \frac{1}{k} \sqrt{\frac{1+\sin \phi_{0}}{2}}\right] .
$$

Combining Eqs. (17), (24) and considering the relation

$$
\cos \phi=\cos \left(\phi-\phi_{0}\right) \cos \phi_{0}-\sin \left(\phi-\phi_{0}\right) \sin \phi_{0},
$$

the integrations of Eqs. (2) result in the profile functions of the adhesive collapsed tube,

$$
\begin{aligned}
x= & a+\frac{2 k}{\alpha}\left(\cos 2 \theta_{0}-\cos 2 \theta\right) \cos \phi_{0} \\
& -\frac{1}{\alpha}\left\{\left[F(k, 2 \theta)-F\left(k, 2 \theta_{0}\right)\right]-2\left[E(k, 2 \theta)-E\left(k, 2 \theta_{0}\right)\right]\right\} \sin \phi_{0}, \\
y= & \frac{2 k}{\alpha}\left(\cos 2 \theta_{0}-\cos 2 \theta\right) \sin \phi_{0} \\
& +\frac{1}{\alpha}\left\{\left[F(k, 2 \theta)-F\left(k, 2 \theta_{0}\right)\right]-2\left[E(k, 2 \theta)-E\left(k, 2 \theta_{0}\right)\right]\right\} \cos \phi_{0},
\end{aligned}
$$

and the arc length as a function of parameters $k$ and $\theta$,

$$
s=a+\frac{1}{\alpha}\left[F(k, 2 \theta)-F\left(k, 2 \theta_{0}\right)\right],
$$

where $E(k, \theta)$ and $F(k, \theta)$ are the first and second kind of incomplete elliptic integrals, respectively:

$$
E(k, \theta)=\int_{0}^{\theta} \sqrt{1-k^{2} \sin ^{2} \theta} \mathrm{d} \theta, \quad F(k, \theta)=\int_{0}^{\theta} \frac{1}{\sqrt{1-k^{2} \sin ^{2} \theta}} \mathrm{d} \theta,
$$

and

$$
\alpha=\sqrt{\frac{C_{1}}{2 D\left(2 k^{2}-1\right)}} .
$$


Substituting Eqs. (28)-(30) into the boundary conditions (4) leads to

$$
\begin{gathered}
a=\pi R-b-\frac{1}{\alpha}\left[F\left(k, 2 \theta_{3}\right)-F\left(k, 2 \theta_{0}\right)\right], \\
b=a+\frac{2 k}{\alpha}\left(\cos 2 \theta_{0}-\cos 2 \theta_{3}\right) \cos \phi_{0} \\
-\frac{1}{\alpha}\left\{\left[F\left(k, 2 \theta_{3}\right)-F\left(k, 2 \theta_{0}\right)\right]-2\left[E\left(k, 2 \theta_{3}\right)-E\left(k, 2 \theta_{0}\right)\right]\right\} \sin \phi_{0}, \\
d_{2}=-\frac{2 k}{\alpha}\left(\cos 2 \theta_{0}-\cos 2 \theta_{3}\right) \sin \phi_{0} \\
-\frac{1}{\alpha}\left\{\left[F\left(k, 2 \theta_{3}\right)-F\left(k, 2 \theta_{0}\right)\right]-2\left[E\left(k, 2 \theta_{3}\right)-E\left(k, 2 \theta_{0}\right)\right]\right\} \cos \phi_{0} .
\end{gathered}
$$

For given values of the equilibrium distance $d_{2}$ and the initial radius of the CNT $R$, it is easy to obtain $k$ from Eq. (35). Then, the lengths $2 a$ and $2 b$ of the flat regions in the adhesive structure of two collapsed tubes can be solved from Eqs. (33) and (34) with the help of Eqs. (31). Thus, the detailed profile of the adhesive structure can be plotted from Eqs. (28)-(30).

When the initial radius of a SWNT reduces, the flat lengths $2 a$ and $2 b$ in the adhesive structure will decrease. When the reducing length $2 a$ equals just right zero, at this moment, $d_{2}=d_{0}$, and the corresponding radius of the SWNT is

$$
\begin{aligned}
R_{\text {IImin }}= & \frac{1}{\pi} \sqrt{\frac{D}{W_{2}}} \sqrt{\frac{2\left(2 k^{2}-1\right)}{3}}\left\{\left(\cos 2 \theta_{0}-\cos 2 \theta_{3}\right) \cos \phi_{0}\right. \\
& \left.+2\left[E\left(k, 2 \theta_{3}\right)-E\left(k, 2 \theta_{0}\right)\right] \sin \phi_{0}+\left[F\left(k, 2 \theta_{3}\right)-F\left(k, 2 \theta_{0}\right)\right]\left(1-\sin \phi_{0}\right)\right\},
\end{aligned}
$$

which we call a critical radius. In this paper, $d_{0}=3.4 \AA$ leads to $R_{\text {IImin }}=13.49 \AA$.

When the initial radius is smaller than the critical one, the length $2 a$ vanishes and the interaction distance $d_{2}$ in a nanotube is not equal to the equilibrium separation $d_{0}$ any more. $d_{2}$ will increase relatively with a decreasing initial radius $R$, and the shape of one of the collapsed SWNT will vary gradually from a pillow shape (partial-collapsed shape) to the deformed one studied in Tang et al. [35].

\section{Comparison of the theoretical predictions and MM simulations}

Molecular mechanics (MM) simulations are performed using the Large-scale Atomic/Molecular Massively Parallel Simulator (LAMMPS) [38] with AIREBO [39] potential (without torsion term) and a fast inertial relaxation engine (FIRE) method [40] to verify the theoretically predicted configurations, as well as to obtain the vdW interaction energies, in which the chiralities $(n, 0)$ and $(n, n)$ for tubes with radii ranging from 0.89 to $3.8 \mathrm{~nm}$ are chosen.

The morphologies of three representatively adhesive collapsed CNTs with different initial radius $R$ are plotted in Figs. 5a-c, in which the three radii are less than, nearly equal to, and larger than the critical radius predicted in Eq. (36), respectively. During our molecular mechanics simulations, the chiralities of the nanotubes do not influence the finally stable configurations, while in Tang et al.'s study [35], the deformations of $(2 n, n)$ and $(n, n)$ tubes are different from those of $(n, 0)$ tubes. Twisting and wrinkling can occur in $(2 n, n)$ and $(n, n)$ tubes. The difference may be induced by the different simulation temperatures. In our molecular mechanics simulation, the absolute temperature is $0 \mathrm{~K}$, while in Tang et al.'s work [35], molecular dynamics simulation was carried out and the absolute temperature that they used was $300 \mathrm{~K}$. In Fig. 5, the solid lines denote our theoretical predictions and the dotted ones represent our molecular simulation results. Clearly, the theoretical predictions agree well with the molecular mechanics (MM) results for cases of SWNTs with radius nearly equaling or larger than the critical one; especially, the lengths of the flat regions are almost identical in both the theoretical and molecular mechanics simulation results. A little deviation exists at the two ends of the adhesive structure, which is due to an assumption of constant energy at the ends in our theoretical analysis. However, in the case of SWNTs with radius less than the critical one, the upper and lower curved parts predicted by the theoretical analysis deviate significantly from the MM simulation result, though the adhesive length at 
(a)
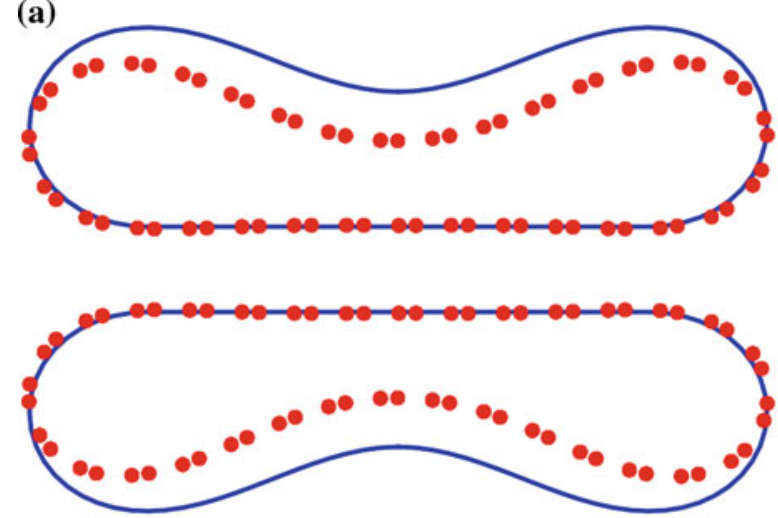

(b)
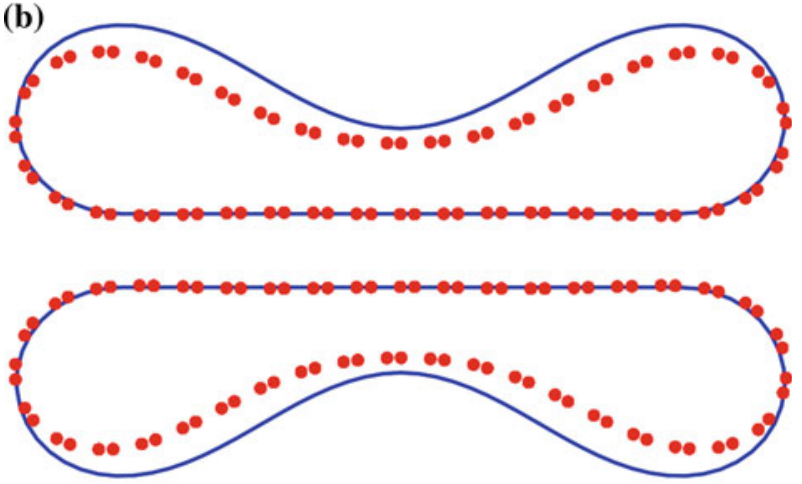

(c)
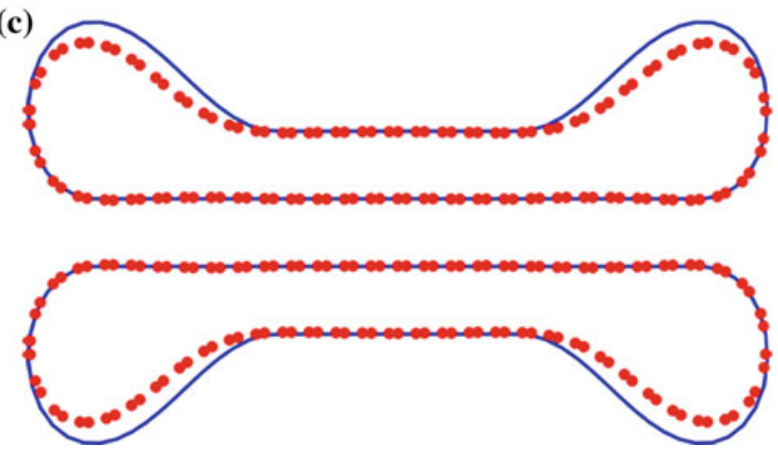

Fig. 2 Three kinds of adhesive collapsed configurations for SWNTs with different initial radius. a Partial-collapsed configuration (pillow-shaped) for the case with an initial radius $(R=10.85 \AA)$ smaller than $R_{\text {IImin }}\left(R_{\text {IImin }}=13.56 \AA\right)$. b Critical collapsed configuration with an initial radius equaling to $R_{\mathrm{IImin}}$. c Collapsed configuration with an initial radius $(R=20.34 \AA)$ larger than $R_{\text {IImin }}$

the interface between two tubes is well consistent with each other. In our theoretical analysis, if the length $2 a$ of the flat region vanishes, the fourth term in Eq. (5) will be zero. The shape of the tube in this case looks like a pillow, and Eq. (5) does not include the inner or inter-interactions of the two tubes except the interaction energy at the interface with length $2 b$. As a result, the inner separations predicted by the theoretical analysis will be larger than those calculated by the MM simulations.

\section{Configuration map for adhering SWNTs}

In fact, there are three types of configurations for adhering identical SWNTs as shown in Fig. 2, where, for the first one, the tube is near a circle when the initial radius of the tube is small; the tube will deform under the inner and inter-interactions of tubes when the radius of the tube becomes large; if the initial radius of the 


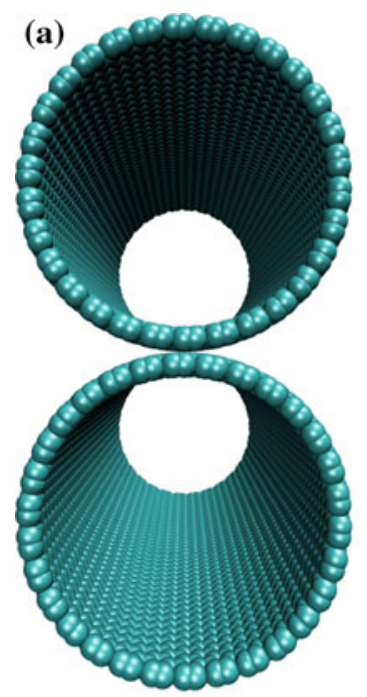

(b)

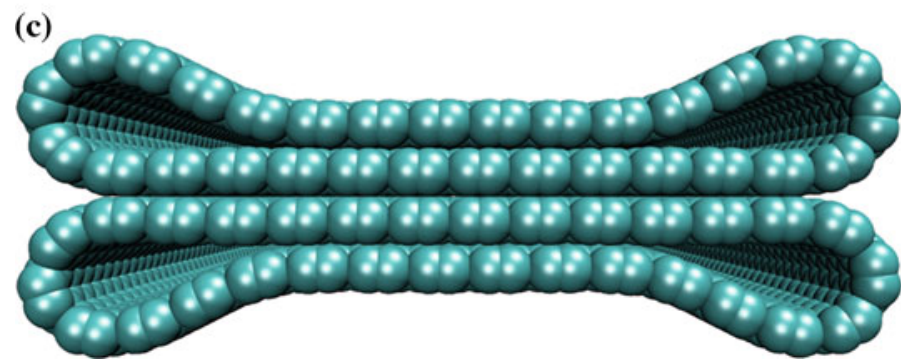

Fig. 3 Three types of configurations formed by two identical SWNTs. a Circular configuration. b Deformed configuration. c Collapsed configuration

tube is large enough, the inner and inter-interactions may render the two adhesive tubes collapse. Three cases are schematically shown in Fig. 2. The first configuration as shown in Fig. 2a has been well studied [32-34]. The second deformed configuration was successfully solved by Tang et al.'s method [35] using continuum mechanics and molecular simulations. In the solution given by Tang et al. [35], three variables and complicated integrals need to be solved. In order to achieve a clear configuration map for all the above three cases, the deformed case is re-studied using the solving technique in the present paper and a simpler solution is given.

\subsection{Solutions to the deformed configuration}

The deformed configuration of two identical carbon nanotubes in adhesive contact with each other is shown in Fig. 3a with a flat adhesive length $2 b$ and an equilibrium distance $d_{0}$. Each tube has an initial radius $R$ and out-of-plane length $L$. Also, a quarter of the structure is considered due to the symmetry as shown in Fig. $3 \mathrm{~b}$. Similar to Eq. (5), the potential energy for the deformed system can be written as

$$
\begin{aligned}
\Pi= & 2 L \int_{a}^{\pi R}\left[D \dot{\phi}^{2}+\lambda_{1}(\dot{x}-\cos \phi)+\lambda_{2}(\dot{y}-\sin \phi)\right] \mathrm{d} s \\
& -2 W_{2} \cdot b \cdot L+4 \pi R L u_{g}+U_{\mathrm{vdW}}^{n c} .
\end{aligned}
$$

A similar method can be used to find the profile functions of the adhesive deformed tube. Details are neglected here, and all the parameters in this case correspond to those in the collapsed case. Then, we have

$$
x(\theta)=b+\frac{1}{\alpha}[2 E(k, \theta)-F(k, \theta)],
$$




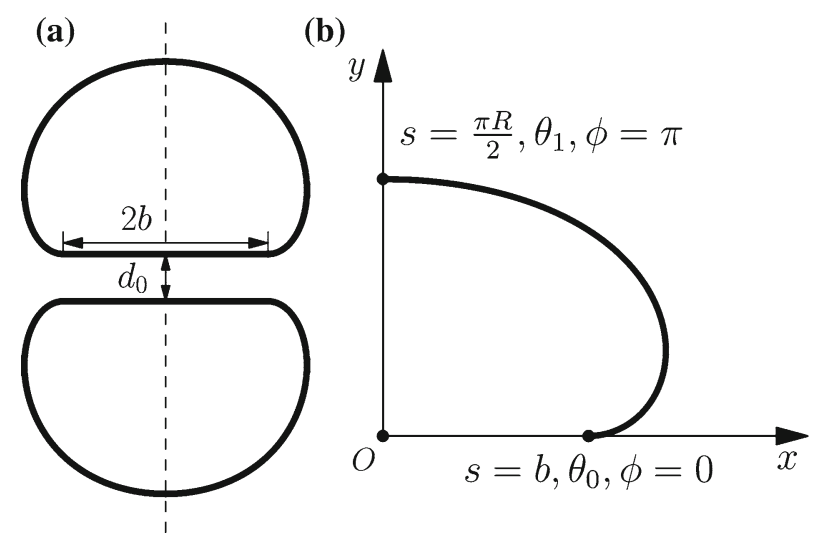

Fig. 4 Deformed configuration of two identical SWNTs. a The interface in adhesive contact with a length $2 b$ and a separated distance $d_{0} ; \mathbf{b}$ a quarter of the adhesion structure for theoretical analysis

$$
\begin{gathered}
y(\theta)=\frac{2 k}{\alpha}(1-\cos \theta), \\
s(\theta)=b+\frac{F(k, \theta)}{\alpha},
\end{gathered}
$$

where $k$ satisfies the equation

$$
4 k\left[F\left(k, \theta_{1}\right)-E\left(k, \theta_{1}\right)\right]=\frac{\pi R}{\sqrt{\frac{D}{W_{2}}}},
$$

and $\theta_{0}=0 \leq \theta \leq \theta_{1}=\arcsin \frac{1}{k}, \alpha=\frac{1}{2 k} \sqrt{\frac{W_{2}}{D}}, b=\pi R-\frac{1}{\alpha} F\left(k, \theta_{1}\right)$.

The contact length $2 b$ will decrease with a decreasing radius $R$. When the contact length vanishes, i.e., $2 b=0$, we obtain another critical radius as

$$
R_{\min }=1.013 \sqrt{\frac{D}{W_{2}}},
$$

which is well consistent with the approximate solution given by Tang et al. [35] $R_{\min }=\sqrt{D / W_{2}}$. When the initial radius of a carbon nanotube is less than the critical radius in Eq. (42), the adhesive configuration of such two nanotubes should be the circular case as shown in Fig. 2a.

\subsection{Comparison between the theoretical predictions and MM calculations}

The potential energy density as a function of the dimensionless tube radius is shown in Fig. 4, in which the theoretical predictions and the MM simulation results are compared with parameters $D=1.3263 \mathrm{eV}$, $W_{1}=19.722 \mathrm{meV} / \AA^{2}$, and $W_{2}=17.872 \mathrm{meV} / \AA^{2}$. Equaling the potential energy for the deformed configuration, i.e., Eq. (5), to that for the collapsed one, i.e., Eq. (37), yields the critical radius $R_{\max }=20.20 \AA$ numerically, above which the collapsed configuration is energetically favored. It is found that both the theoretical and MM calculation results are consistent with each other. It can infer that when the radius of a tube $R$ is smaller than $R_{\min }$, the shape of the tubes in the adhesion system is circular. When the radius of a tube lies in the region $R_{\min }<R<R_{\max }$, both the deformed and the collapsed configurations exist, but the potential energy of the collapsed configuration is higher than the deformed one, which means the deformed configuration is more stable than the collapsed one. For $R>R_{\max }$, the potential energy of the deformed configuration is higher than the collapsed one, and thus, the collapsed configuration is more stable at this region. One may note that there is another region $R_{\min }<R<R_{\mathrm{IImin}}$, in which one data in the MM simulation for the collapsed case drops onto the curve for the deformed case. As mentioned above, $R_{\text {IImin }}$ is a critical radius, at which the contact 


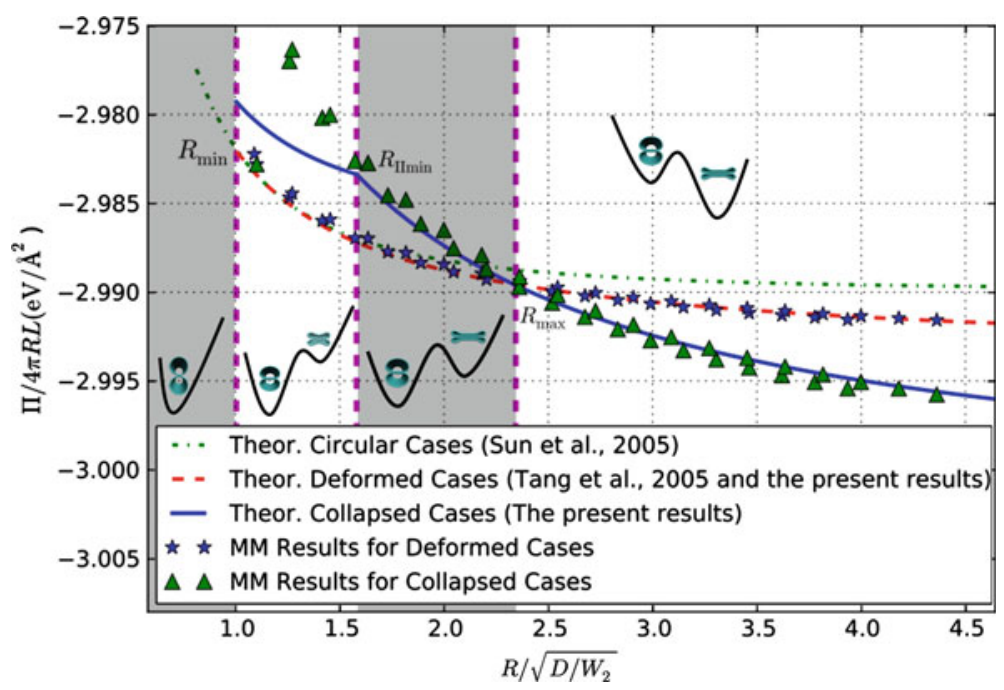

Fig. 5 The potential energy density $\Pi / 4 \pi R L$ as a function of the dimensionless radius $R / \sqrt{D / W_{2}}$, in which three types of adhesive configurations are separated by two critical radii $R_{\min }$ and $R_{\max } . R_{\mathrm{IImin}}$ is a special one in order to distinguish the partial-collapsed configuration from the collapsed one

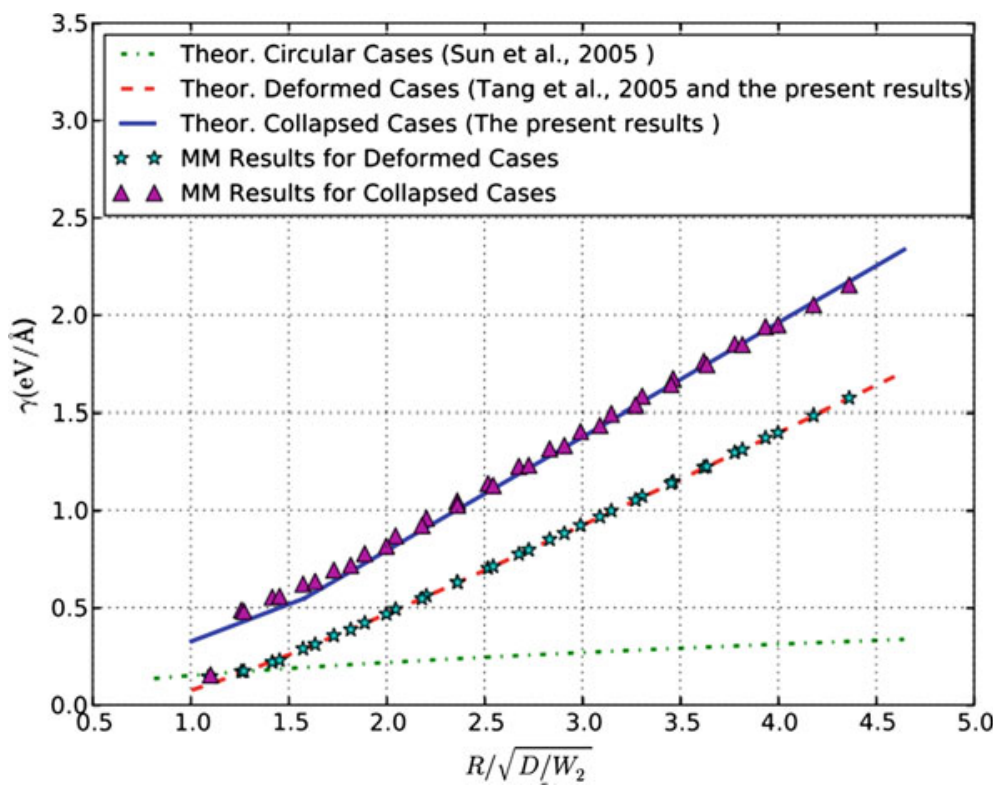

Fig. 6 Comparison of theoretical predictions and MM simulation results of the adhesion energy as a function of the dimensionless radius of SWNTs for three types of adhesion structures

width in a self-collapsed tube vanishes, i.e., $a=0$, and below which the partial-collapsed configuration may exist. However, comparison between the potential energies of the deformed case and the partial-collapsed one exhibits that the deformed configuration is more stable in the region $R_{\min }<R<R_{\mathrm{IImin}}$.

The adhesion energy $\gamma$ as a function of $R / \sqrt{D / W_{2}}$ is plotted in Fig. 6, where MM results are shown for comparison, and the adhesion energies $\gamma$ for the three cases are calculated as follows:

(1) In the case of the circular configuration, the adhesion energy is $\gamma=\phi\left(R, R, 2 R+d_{0}\right)$ [32].

(2) In the case of the deformed configuration, the adhesion energy can be formulated as $\gamma=2 W_{2} \cdot b+$ const. and the constant can be found from the molecular simulation as $-0.076 \mathrm{eV} / \AA$.

(3) In the case of the collapsed configuration, the adhesion energy can be expressed as $\gamma=4\left(W_{1}-W_{2}\right)$. $a+2 W_{2} \cdot b+$ const. and the constant is $-0.067 \mathrm{eV} / \AA$ found from the molecular simulation. 
From Fig. 6, it is clearly seen that the adhesion energy of the collapsed configuration is much larger than the other two cases. This means that tube arrays combined by collapsed tubes should have strong inner interface strength and should be more useful in the applications of composites as reinforced fibers.

\section{Conclusions}

Inspired by experimental observations, a theoretical model for adhesion structures between two identically collapsed carbon nanotubes is established and analyzed under the framework of the continuum mechanics. The detailed shape function for the adhesion structure is achieved analytically. Combining the results for the circular-shaped configuration and the deformed one, as well as our theoretical predictions and molecular mechanics simulations for the present collapsed configuration, shows that such three types of configurations are separated by two critical radii of the nanotubes. Furthermore, the adhesion energies for three cases demonstrate that the collapsed configuration possesses the highest one and should be more useful in applications of carbon nanotube reinforced composites and nano-electromechanical system fields due to a strong inner interface.

Acknowledgments The work reported here is supported by NSFC through Grants \#10972220, \#11125211, \#11021262 and the Nano-project (2012CB937500). CZ thanks Dr. Chao Wang (THU) and Prof. Chenggang Zhou (CUG) for helpful discussions. The molecular simulations are carried out at Supercomputing Center of Chinese Academy of Sciences.

\section{References}

1. Glassmaker, N.J., Hui, C.Y.: Elastica solution for a nanotube formed by self-adhesion of a folded thin film. J. Appl. Phys. 96, 3429 (2004)

2. Ajayan, P.M., Stephan, O., Colliex, C., Trauth, D.: Aligned carbon nanotube arrays formed by cutting a polymer resinnanotube composite. Science 265, 1212-1214 (1994)

3. Chan, S.-P., Yim, W.-L., Gong, X., Liu, Z.-F.: Carbon nanotube bundles under high pressure: transformation to low-symmetry structures. Phys. Rev. B 68, 075404 (2003)

4. Franklin, N.R., Wang, Q., Tombler, T.W., Javey, A., Shim, M., Dai, H.: Integration of suspended carbon nanotube arrays into electronic devices and electromechanical systems. Appl. Phys. Lett. 81, 913 (2002)

5. Liu, Z., Jiao, L., Yao, Y., Xian, X., Zhang, J.: Aligned, ultralong single-walled carbon nanotubes: from synthesis, sorting, to electronic devices. Adv. Mater. 22, 2285-2310 (2010)

6. Tersoff, J., Ruoff, R.: Structural properties of a carbon-nanotube crystal. Phys. Rev. Lett. 73, 676-679 (1994)

7. Ruoff, R.S., Tersoff, J., Lorents, D.C., Subramoney, S., Chan, B.: Radial deformation of carbon nanotubes by van der Waals forces. Nature 364, 514-516 (1993)

8. Chopra, N.G., Benedict, L.X., Crespi, V.H., Cohen, M.L., Louie, S.G., Zettl, A.: Fully collapsed carbon nanotubes. Nature 377, 135-138 (1995)

9. Yu, M.-F., Dyer, M.J., Ruoff, R.S.: Structure and mechanical flexibility of carbon nanotube ribbons: an atomic-force microscopy study. J. Appl. Phys. 89, 4554 (2001)

10. Yu, M.-F., Kowalewski, T., Ruoff, R.: Structural analysis of collapsed, and twisted and collapsed, multiwalled carbon nanotubes by atomic force microscopy. Phys. Rev. Lett. 86, 87-90 (2001)

11. Yu, M.: Strength and breaking mechanism of multiwalled carbon nanotubes under tensile load. Science 287, 637-640 (2000)

12. Liu, S., Yue, J., Wehmschulte, R.J.: Large thick flattened carbon nanotubes. Nano Lett. 2, 1439-1442 (2002)

13. Zhang, C., Bets, K., Lee, S.S., Sun, Z., Mirri, F., Colvin, V.L., Yakobson, B.I., Tour, J.M., Hauge, R.H.: Closed-edged graphene nanoribbons from large-diameter collapsed nanotubes. Acs Nano 6, 6023-6032 (2012)

14. Wang, R., Hao, Y., Wang, Z., Gong, H., Thong, J.T.L.: Large-diameter graphene nanotubes synthesized using Ni nanowire templates. Nano Lett. 10, 4844-4850 (2010)

15. Alvarez, N.T., Li, F., Pint, C.L., Mayo, J.T., Fisher, E.Z., James, M., Colvin, V.L., Hauge, R.H., Tour, J.M.: Uniform large diameter carbon nanotubes in vertical arrays from premade near-monodisperse nanoparticles. Chem. Mater. 23, 3466$3475(2011)$

16. Queipo, P., Nasibulin, A.G., Shandakov, S.D., Jiang, H., Gonzalez, D., Kauppinen, E.I.: CVD synthesis and radial deformations of large diameter single-walled CNTs. Curr. Appl. Phys. 9, 301-305 (2009)

17. Motta, M., Moisala, A., Kinloch, I.A.A., Windle, A.H.H.: High performance fibres from "dog bone" carbon nanotubes. Adv. Mater. 19, 3721-3726 (2007)

18. Koziol, K., Vilatela, J., Moisala, A., Motta, M., Cunniff, P., Sennett, M., Windle, A.: High-performance carbon nanotube fiber. Science 318, 1892-1895 (2007)

19. Vilatela, J.J., Khare, R., Windle, A.H.: The hierarchical structure and properties of multifunctional carbon nanotube fibre composites. Carbon 50, 1227-1234 (2012)

20. Byrne, M.T., Gun'ko, Y.K.: Recent advances in research on carbon nanotube-polymer composites. Adv. Mater. 22, 1672$1688(2010)$

21. Cheng, Q., Li, M., Jiang, L., Tang, Z.: Bioinspired layered composites based on flattened double-walled carbon nanotubes. Adv. Mater. 24, 1838-1843 (2012)

22. Senga, R., Hirahara, K., Nakayama, Y.: Nanotorsional actuator using transition between flattened and tubular states in carbon nanotubes. Appl. Phys. Lett. 100, 083110 (2012) 
23. Giusca, C., Tison, Y., Silva, S.: Atomic and electronic structure in collapsed carbon nanotubes evidenced by scanning tunneling microscopy. Phys. Rev. B. 76, 1-6 (2007)

24. Tombler, T., Zhou, C., Alexseyev, L., Kong, J., Dai, H., Liu, L., Jayanthi, C., Tang, M., Wu, S.: Reversible electromechanical characteristics of carbon nanotubes under local-probe manipulation. Nature 405, 769-772 (2000)

25. Gómez-Navarro, C., Sáenz, J., Gómez-Herrero, J.: Conductance oscillations in squashed carbon nanotubes. Phys. Rev. Lett. 96, 076803 (2006)

26. Giusca, C.E., Tison, Y., Silva, S.R.P.: Evidence for metal-semiconductor transitions in twisted and collapsed double-walled carbon nanotubes by scanning tunneling microscopy. Nano Lett. 8, 3350-3356 (2008)

27. Yu, W.J., Chae, S.H., Perello, D., Lee, S.Y., Han, G.H., Yun, M., Lee, Y.H.: Synthesis of edge-closed graphene ribbons with enhanced conductivity. Acs Nano 4, 5480-5486 (2010)

28. Pugno, N.M.: The design of self-collapsed super-strong nanotube bundles. J. Mech. Phys. Solids 58, 1397-1410 (2010)

29. Zhang, X., Li, Q.: Enhancement of friction between carbon nanotubes: an efficient strategy to strengthen fibers. Acs Nano 4, 312-316 (2010)

30. Chen, B., Gao, M., Zuo, J.M., Qu, S., Liu, B., Huang, Y.: Binding energy of parallel carbon nanotubes. Appl. Phys. Lett. 83, 3570 (2003)

31. Girifalco, L., Hodak, M., Lee, R.: Carbon nanotubes, buckyballs, ropes, and a universal graphitic potential. Phys. Rev. B 62, 13104-13110 (2000)

32. Sun, C.-H., Yin, L.-C., Li, F., Lu, G.-Q., Cheng, H.-M.: Van der Waals interactions between two parallel infinitely long single-walled nanotubes. Chem. Phys. Lett. 403, 343-346 (2005)

33. Zhbanov, A.I., Pogorelov, E.G., Chang, Y.-C.: Van der Waals interaction between two crossed carbon nanotubes. Acs Nano 4, 5937-5945 (2010)

34. Pogorelov, E.G., Zhbanov, A.I., Chang, Y.-C., Yang, S.: Universal curves for the van der Waals interaction between singlewalled carbon nanotubes. Langmuir 28, 1276-1282 (2012)

35. Tang, T., Jagota, A., Hui, C.-Y.: Adhesion between single-walled carbon nanotubes. J. Appl. Phys. 97, 074304 (2005)

36. Liu, J.: Explicit solutions for a SWCNT collapse. Arch. Appl. Mech. 82, 767-776 (2011)

37. Lu, Q., Arroyo, M., Huang, R.: Elastic bending modulus of monolayer graphene. J. Phys. D Appl. Phys. 42, 102002 (2009)

38. Plimpton, S.: Fast parallel algorithms for short-range molecular dynamics. J. Comput. Phys. 117, 1-19 (1995)

39. Brenner, D.W., Shenderova, O.A., Harrison, J.A., Stuart, S.J., Ni, B., Sinnott, S.B.: A second-generation reactive empirical bond order (REBO) potential energy expression for hydrocarbons. J. Phys. Condens. Matter. 14, 783-802 (2002)

40. Bitzek, E., Koskinen, P., Gähler, F., Moseler, M., Gumbsch, P.: Structural relaxation made simple. Phys. Rev. Lett. 97, $1-4(2006)$ 